\title{
Deletion of all three MAP kinase genes results in severe defects in stress responses and pathogenesis in Fusarium graminearum
}

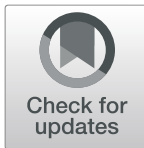

Jingyi Ren', Yuhan Zhang ${ }^{1}$, Yuhua Wang ${ }^{1}$, Chengliang Li ', Zhuyun Bian², Xue Zhang ${ }^{1,2}$, Huiquan Liu', Jin-Rong $\mathrm{Xu}^{2^{*}}$ (D) and Cong Jiang ${ }^{1 *}$ (D)

\begin{abstract}
Mitogen-activated protein kinase (MAPK) cascades are activated by external stimuli and convert signals to cellular changes. Individual MAPKs have been characterized in a number of plant pathogenic fungi for their roles in pathogenesis and responses to biotic or abiotic stresses. However, mutants deleted of all the MAPK genes have not been reported in filamentous fungi. To determine the MAPK-less effects in a fungal pathogen, in this study we generated and characterized mutants deleted of all three MAPK genes in the wheat scab fungus Fusarium graminearum. The Gpmk1 mgv1 Fghog1 triple mutants had severe growth defects and was non-pathogenic. It was defective in infection cushion formation and DON production. Conidiation was reduced in the triple mutant, which often produced elongated conidia with more septa than the wild-type conidia. The triple mutant was blocked in sexual reproduction due to the loss of female fertility. Lack of any MAPKs resulted in an increased sensitivity to various abiotic stress including cell wall, osmotic, oxidative stresses, and phytoalexins, which are likely related to the defects of the triple mutant in environmental adaptation and plant infection. The triple mutant also had increased sensitivity to the biocontrol bacterium Bacillus velezensis and fungus Clonostachys rosea. In co-incubation assays with B. velezensis, the Gpmk1 mgv1 Fghog1 mutant had more severe growth limitation than the wild type and was defective in conidium germination and germ tube growth. In confrontation assays, the triple mutant was defective in defending against mycoparasitic activities of C. rosea and the latter could grow over the mutant but not wildtype F. graminearum. RNA-seq and metabolomics analyses showed that the MAPK triple mutant was altered in the expression of many ATP-binding cassette (ABC) and major facilitator superfamily (MFS) transporter genes and the accumulation of metabolites related to arachidonic acid, linoleic acid, and alpha-linolenic acid metabolisms. Overall, as the first study on mutants deleted of all three MAPKs in fungal pathogens, our results showed that although MAPKs are not essential for growth and asexual reproduction, the Gpmk1 mgv1 Fghog1 triple mutant was blocked in plant infection and sexual reproductions. It also had severe defects in responses to various abiotic stresses and bacterial- or fungal-fungal interactions.
\end{abstract}

Keywords: Abiotic stresses, Fungal-bacterial interaction, Gibberella zeae, Mycoparasitism, Signal transduction

\footnotetext{
* Correspondence: jinrong@purdue.edu; cjiang@nwafu.edu.cn

${ }^{2}$ Department of Botany and Plant Pathology, Purdue University, West

Lafayette, IN 47907, USA

1 State Key Laboratory of Crop Stress Biology for Arid Areas and

NWAFU-Purdue Joint Research Center, College of Plant Protection, Northwest

A\&F University, Yangling 712100, Shaanxi, China
}

(c) The Author(s). 2022 Open Access This article is licensed under a Creative Commons Attribution 4.0 International License, which permits use, sharing, adaptation, distribution and reproduction in any medium or format, as long as you give appropriate credit to the original author(s) and the source, provide a link to the Creative Commons licence, and indicate if changes were made. The images or other third party material in this article are included in the article's Creative Commons licence, unless indicated otherwise in a credit line to the material. If material is not included in the article's Creative Commons licence and your intended use is not permitted by statutory regulation or exceeds the permitted use, you will need to obtain permission directly from the copyright holder. To view a copy of this licence, visit http://creativecommons.org/licenses/by/4.0/. 


\section{Introduction}

The homothallic ascomycete Fusarium graminearum is a causal agent of Fusarium Head Blight (FHB), a destructive disease of wheat, barley, and other grain cereals worldwide (Harris et al., 2016). Under favorable environmental conditions, infection of wheat or barley heads by $F$. graminearum often results in severe yield losses, grain quality reduction, and contamination of mycotoxins such as deoxynivalenol (DON) and zearalenone (Audenaert et al., 2013; Goswami and Kistler 2004). The fungus overwinters and forms perithecia on infected plant debris. Ascospores forcefully released from perithecia are the primary inoculum and F. graminearum infection can occur from anthesis to kernel filling. DON is an important virulence factor in this pathogen and TRI genes responsible for DON biosynthesis are expressed in infection cushions (Boenisch and Schafer 2011). For asexual reproduction, it produces macroconidia or conidia, which also are infectious and important for spreading infection and colonization of plant tissues. To survive in the nature and infect wheat or barley spikelets, $F$. graminearum must be able to sense various environmental and plant signals for properly regulating various developmental and infection production as well as DON production (Dilks et al., 2019; Jiang et al., 2019).

Like in other eukaryotic organisms, mitogen-activated protein (MAP) kinases play important roles in activating cellular responses to extracellular signals, including host and environmental stimuli in plant pathogenic fungi (Jiang et al., 2018a). In general, signals sensed by specific receptors are relayed to MAP kinase (MAPK) cascades to activate downstream transcription factors for regulating changes in gene expression ( $\mathrm{Gu}$ et al., 2015; Jiang et al. 2019; Wang et al., 2015; Yun et al., 2014). Unlike the budding yeast Saccharomyces cerevisiae that has five MAPK genes involved in regulating pheromone response, filamentation, cell wall integrity, osmoregulation, and ascospore formation (Posas et al., 1998), F. graminearum and most ascomycetous phytopathogenic fungi have only three MAPKs, which are orthologous to yeast Fus3/Kss1, Slt2, and Hog1 (Li et al., 2012). The first MAPK gene characterized in F. graminearum is MGV1, an ortholog of yeast $S L T 2$ cell wall integrity MAPK and MPS1 of the rice blast fungus Magnaporthe oryzae (Bermejo et al., 2008; Hou et al., 2002; Xu et al., 1998). The mgv1 mutant was significantly reduced in growth rate and hypersensitive to cell wall stress. $\mathrm{Mu}$ tants deleted of $M G V 1$ or its upstream MAPK kinase (MEK) and MEK kinase (MEKK) genes are nonpathogenic and sterile, and have severe cell wall defects (Hou et al. 2002; Wang et al., 2011; Yun et al. 2014). Deletion of GPMK1 or MAP 1, an ortholog of $M$. oryzae PMK1 also results in defects in DON production and sexual reproduction (Jenczmionka et al.,
2003; Urban et al., 2003). Gpmk1 in F. graminearum also regulates the early induction of extracellular endoglucanase, xylanolytic, and proteolytic activities (Jenczmionka and Schafer 2005). Unlike the orthologs of $M G V 1$ and GPMK1 that are essential for plant infection in general, the HOG pathway has a conserved role in osmoregulation but species-specific functions in pathogenesis in fungal pathogens (Jiang et al. 2018a; Zhang et al., 2021). Whereas its ortholog in M. oryzae is dispensable for virulence, FgHOG1 is essential for plant infection in F. graminearum. Besides regulating responses to hyperosmotic and oxidative stresses and resistance against fludioxonil, the FgHog1 MAPK pathway is also involved in the regulation of DON biosynthesis and sexual reproduction (Wang et al. 2011; Zheng et al., 2012). Interestingly, null mutations in FgHOG1 partially rescue the defect of the mgv1 mutant in growth and cell wall integrity but not pathogenesis (Ren et al., 2019).

Although individual MAPK genes have been characterized for their regulatory roles in pathogenesis, development, and stress responses in a number of plant pathogenic fungi, to date, there is no report on mutants deleted of all three MAPK genes in any fungal pathogen. In F. graminearum, the Gpmk1, Mgv1, and FgHog1 MAPKs have distinct functions but they are all important for plant infection, DON production, and sexual reproduction, suggesting their overlapping roles in pathogenesis, development, and secondary metabolism. To further characterized the relationship among these three MAPK pathways, in this study we generated and characterized mutants deleted of GPMK1, MGV1, and FgHOG1. The Gpmk1 mgv1 Fghog1 triple deletion mutant was viable but had severe growth defects. It was non-pathogenic and female sterile. Besides increased sensitivity to a variety of abiotic stresses, the triple mutant had increased sensitivity to biocontrol agents Bacillus velezensis and Clonostachys rosea. To our knowledge, this is the first study on mutants deleted of all three MAPKs in fungal pathogens, and our results showed that although MAPKs are not essential for growth and asexual reproduction, the Gpmk1 mgv1 Fghog1 triple mutant was blocked in plant infection and sexual reproductions. It also had severe defects in responses to various abiotic stresses and bacterial- or fungal-fungal interactions.

\section{Results}

The Gpmk1 mgv1 Fghog1 triple mutant is defective in hyphal growth and development

To generate the Gpmk1 mgv1 Fghog1 triple mutant in F. graminearum, we first generated the Gpmk1 mgv1 double mutant by deleting the MGV1 gene in the Gpmk1 mutant (Wang et al. 2011). The resulting double 
mutant was used to generate the triple mutant by transforming the FgHOG1 gene replacement construct into the Gpmk1 mgv1 mutant (Table S1). Deletion of MGV1 and FgHOG1 in the Gpmk1 mgv1 Fghog1 triple mutant was verified by PCR with anchor primers (Table S1, Fig. S1). Three triple mutant strains were identified. All three of them had the same phenotype although only data for one of them were presented below. Unlike the Gpmk1 and Fghog1 mutants, the mgv1 mutant had severe growth defects (Wang et al. 2011). Interestingly, in comparison with the Gpmk1 mgv1 double mutant, the growth rate was slightly increased in the triple mutant. Nevertheless, the triple mutant still grew slower than the wild type and Gpmk1 mutant (Fig. 1a; Table 1). Furthermore, aerial hyphae produced by the triple mutant was reduced in surface hydrophobicity, likely caused by deletion of FgHOG1 (Zheng et al. 2012) (Fig. S2). In comparison with the wild type, the triple mutant produced wavy germ tubes and hyphae (Fig. 1b), which was not observed in the Gpmk1 and Gpmk1 mgv1 mutants (Fig. $1 b)$, suggesting an overlapping function of three MAPKs in hyphal growth. These results indicate that deletion of all three MAPKs is not lethal in F. graminearum but resulted in defects in hyphal growth and colony surface properties.

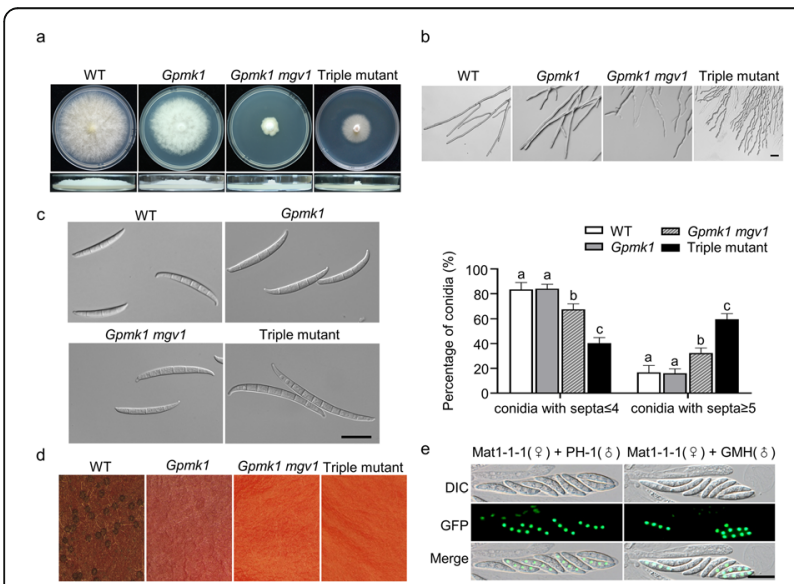

Fig. 1 Assay for the roles of MAPKs in hyphal growth, conidiation, and sexual reproduction. a. 4-day-old CM cultures and growth rate of the wild type (WT) and Gpmk1, Gpmk1 mgv1 double, and Gpmk1 mgv1 Fghog1 triple mutants. $\mathbf{b}$. Hyphal tip growth and branching patterns of the indicated strains on 1/2 CM plates. Scale bars, $50 \mu \mathrm{m}$. c. Conidial morphology of the indicated strains. Scale bars, $20 \mu \mathrm{m}$. Percentage of conidia with $\leq 4$ and $\geq 5$ septa in the indicated strains. d. Self-mating cultures of the indicated strains were examined for perithecia and cirrhi at 7 days post-fertilization. e. Asci and ascospores from the out-crosses between the mat1-1 H1-GFP strain (female) and the wild type or triple mutant (male) at $7 \mathrm{dpf}$ were examined by DIC and epifluorescence microscopy. Scale bars, $20 \mu \mathrm{m}$. Mean and standard deviation were estimated with data from at least three $(n=3)$ independent biological replicates. Different letters indicate significant differences based on ANOVA analysis followed by Duncan's multiple range test $(P=0.05)$
Table 1 Defects of the Gmpk1, Gpmk1 mgv1 and Gpmk1 mgv1 Fghog1 mutants in growth, conidiation, and plant infection

\begin{tabular}{llll}
\hline & $\begin{array}{l}\text { Growth rate } \\
(\mathbf{m m} / \text { day })^{\mathrm{A}}\end{array}$ & $\begin{array}{l}\text { Conidiation } \\
\left(\times 10^{4} / \mathbf{m l}^{\mathbf{B}}\right.\end{array}$ & Disease index \\
\hline WT & $9.24 \pm 0.36^{\mathrm{a}}$ & $113.61 \pm 10.15^{\mathrm{a}}$ & $9.9 \pm 2.67^{\mathrm{a}}$ \\
Gpmk1 & $7.28 \pm 0.15^{\mathrm{b}}$ & $46.89 \pm 7.17^{\mathrm{b}}$ & $\mathrm{NA}$ \\
Gpmk1 mgv1 & $2.58 \pm 0.15^{\mathrm{d}}$ & $48.11 \pm 18.17^{\mathrm{b}}$ & $\mathrm{NA}$ \\
Triple mutant & $3.72 \pm 0.26^{\mathrm{c}}$ & $47.61 \pm 10.80^{\mathrm{b}}$ & $0^{\mathrm{b}}$ \\
\hline
\end{tabular}

A. Average daily extension in colony radius was measured on CM plates. Mean and standard deviation were calculated from at least three independent measurements

B. Conidiation in 5-day-old CMC cultures

C. Disease index was rated by the number of symptomatic spikelets per wheat head at 14 days post-inoculation (dpi)

Data were analyzed with Duncan's pairwise comparison. Different letters mark statistically significant differences $(P=0.05)$

NA. not analyzed

When assayed for conidiation in liquid CMC medium, the Gpmk1 mgv1 Fghog1 mutant was reduced 2-fold in conidiation compared to the wild type, which was similar to the Gpmk1 and Gmpk1 mgv1 mutant (Table 1), indicating the importance of Gpmk1 in conidiation. In addition, conidia of the triple mutant were longer and had more septa than those of the wild type (Fig. 1c). Whereas majority of the wild-type conidia had three or four septa, more than half of the Gpmk1 mgv1 Fghog1 conidia had five or more septa (Fig. 1c). Conidia of the Gpmk1 mgv1 double mutant tended to be longer than the wild type and Gpmk1 mutant, but still shorter and had less septa than those of the triple mutant (Fig. 1c). Because deletion of individual MAPK genes is not known to affect conidium morphology in $F$. graminearum (Hou et al. 2002; Jenczmionka and Schafer 2005; Ren et al. 2019; Wang et al. 2011; Zheng et al. 2012), Gpmk1, Mgv1, and FgHog1 likely hare overlapping or redundant roles in conidiogenesis and conidium morphology.

On self-mating carrot agar cultures, the triple mutant was sterile (Fig. 1d). Whereas abundant perithecia were formed by the wild type at 7 days post-fertilization (dpf), the Gpmk1 mgv1 Fghog1 mutant failed to produce perithecia under the same conditions (Fig. 1d). We then conducted outcrosses with the Gpmk1 mgv1 Fghog1 triple mutant to determine whether it is defective in male or female fertility. When the triple mutant was used as the male to fertilize a mat1-1 H1-GFP strain, fertile perithecia with normal asci and ascospores were observed at $7 \mathrm{dpf}$ (Fig. 1e). Four of the eight ascospores in each ascus had GFP signals in the nucleus, suggesting the segregation of H1-GFP in the progeny (Fig. 1e). Therefore, deletion of all the MAPKs has no effect on male fertility but results in the loss of female fertility in selfing in F. graminearum.

\section{Deletion of all three MAPK genes results in the loss of pathogenicity and DON production}

In infection assays with flowering wheat heads, the Gpmk1 mgv1 Fghog1 deletion mutant failed to cause 
symptoms on the inoculated kernels and were nonpathogenic to wheat (Table 1; Fig. 2a). In infection assays with corn silks, the triple mutant caused very limited discoloration at the inoculation site. Extensive discoloration was observed only in samples inoculated with the wild type (Fig. 2b).

F. graminearum is known to form infection cushions for plant penetration. When examined by scanning electron microscopy (SEM), abundant infection cushions were formed by the wild type on wheat lemma at 2 days post-inoculation (dpi) (Fig. 2c). However, typical infection cushions were rarely formed by the triple mutant (Fig. 2c). In infection assays with wheat coleoptiles, extensive invasive growth and necrosis were only observed in samples inoculated with the wild type. In samples inoculated with Gpmk1 mgv1 Fghog1 conidia, invasive hyphae were often restricted to the initial penetrated coleoptile cells (Fig. 2d). Extensive spreading of invasive hyphae and necrosis beyond the wounding inoculation site were rarely observed in samples inoculated with the triple mutant, suggesting the importance of these MAPKs during infection cushion formation and infectious growth.

Because DON is an important virulence factor, we assayed DON production during plant infection. In wheat kernels inoculated with the wild type and collected at $14 \mathrm{dpi}$, over $1000 \mathrm{ppm}$ DON was detected. Under the same conditions, DON was not detectable in

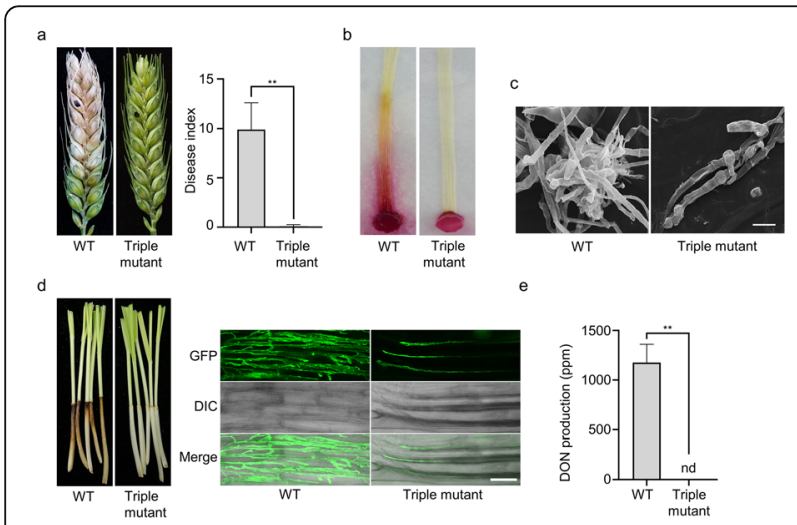

Fig. 2 Defects of the gmpk1 mgv1 Fghog1 triple mutant in plant infection. a. Typical wheat heads of cultivar XiaoYan 22 dropinoculated with conidia of the wild type and Gpmk1 mgv1 Fghog 1 triple mutant were examined for FHB symptoms and measured for disease index at $14 \mathrm{dpi}$. Black dots mark the inoculated spikelets. b. Corn silks inoculated with the wild type and triple mutant were examined at 5 dpi. c. Formation of infection cushions on lemma were examined by SEM. Scale bars, $10 \mu \mathrm{m}$. $\mathbf{d}$. Infected coleoptiles were examined for necrosis and invasive hyphae after staining with Alexa Fluor 488 . Scale bars, $50 \mu \mathrm{m}$. e. DON production in the inoculated wheat kernels sampled at $14 \mathrm{dpi}$. Mean and standard deviation were estimated with data from at least three $(n=3)$ independent biological replicates. The asterisk indicates significant differences based on Student's $t$-test. ${ }^{*} P<0.01$ samples inoculated with the Gpmk1 mgv1 Fghog1 mutant (Fig. 2e). Taken together, these results indicated that plant infection and DON biosynthesis are blocked when all three MAPKs are deleted.

\section{The triple mutant is hypersensitive to cell wall, osmotic, and oxidative stresses}

The Mgv1 MAPK is known to have a conserved role in cell wall integrity (CWI) and deletion of FgHOG1 partially suppress the hypersensitivity of $m g v 1$ mutant to cell wall stress (Ren et al. 2019). When cultured on CM medium with $200 \mu \mathrm{g} / \mathrm{ml}$ Congo red (CR), a commonly used cell wall stressor, the Gpmk1 mgv1 Fghog1 triple mutant displayed similar sensitivity as the mgv1 Fghog1 double mutant, but grew better than the mgv1 mutant (Fig. 3a). Therefore, Gpmk1 is likely dispensable for cell wall stress responses when Mgv1 and FgHog1 are deleted.

In general, fungal osmotic regulation is mainly regulated by the well-conserved HOG MAPK pathway although the other two MAPKs may have minor effects in regulating responses to hyperosmotic stresses in some fungi (Zhang et al. 2021). In the presence of $0.7 \mathrm{M} \mathrm{NaCl}$, germ tubes had subapical swelling in the Fghog1 mutant and apical swelling in the triple mutant. Nevertheless, irregular swellings were not observed in the Gpmk1, Gpmk1 mgv1, and mgv1 Fghog1 mutants (Fig. 3b). These results indicated that Gpmk1 is not important for osmoregulation in the wild type but it may have opposite roles with Mgv1 on responses to hyperosmotic stress in the absence of FgHog1.

In comparison with the wild type, the triple MAPK mutant also displayed increased sensitivity to oxidative stress and only formed compact colonies with limited growth in the presence of $0.05 \% \mathrm{H}_{2} \mathrm{O}_{2}$ (Fig. 3c). Over 90\% of Gpmk1 mgv1 Fghog1 conidia failed to germinate after incubation for $6 \mathrm{~h}$ in the presence of $\mathrm{H}_{2} \mathrm{O}_{2}$, which had only a minor effect on conidium germination in the wild type (Fig. 3d). When cultured for $12 \mathrm{~h}$ in YEPD liquid medium with $\mathrm{H}_{2} \mathrm{O}_{2}$, more severe defects in germ tube growth were observed in the triple mutant than that in the wild type (Fig. 3e).

Brassinin is a phytoalexin with antifungal activities against a number of fungal pathogens (Sellam et al., 2007). The Gpmk1 mgv1 Fghog1 triple mutant also was hypersensitive to brassinin (Fig. 3f). The presence of $250 \mu \mathrm{M}$ brassinin inhibited not only colonial growth but also conidium germination significantly in the triple mutant in comparison with the wild type (Fig. 3f; g). Deletion of GPMK1, MGV1, or FgHOG1 individually also resulted in hypersensitivity to brassinin in colonial growth, but the Gpmk1 mutant had a higher level of tolerance against brassinin than the other two MAPK mutants (Fig. 3f). In contrast, the $m g v 1$ mutant but not the 


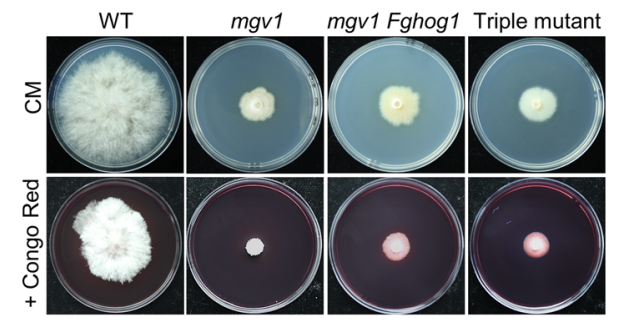

C
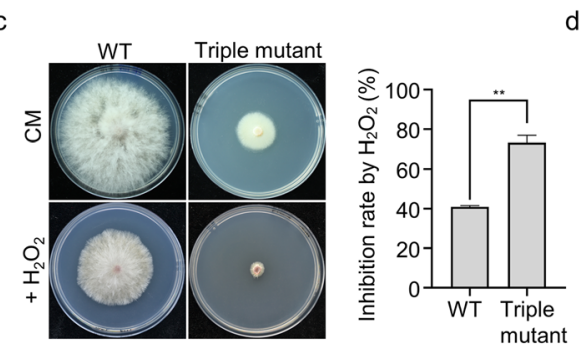

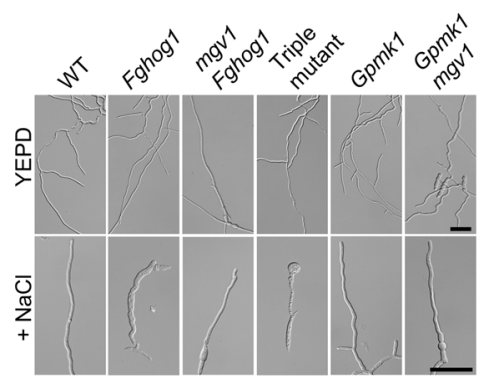

e

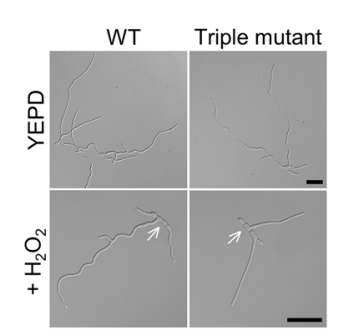

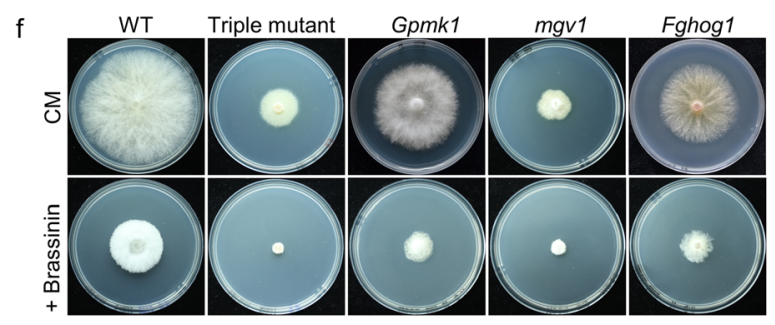
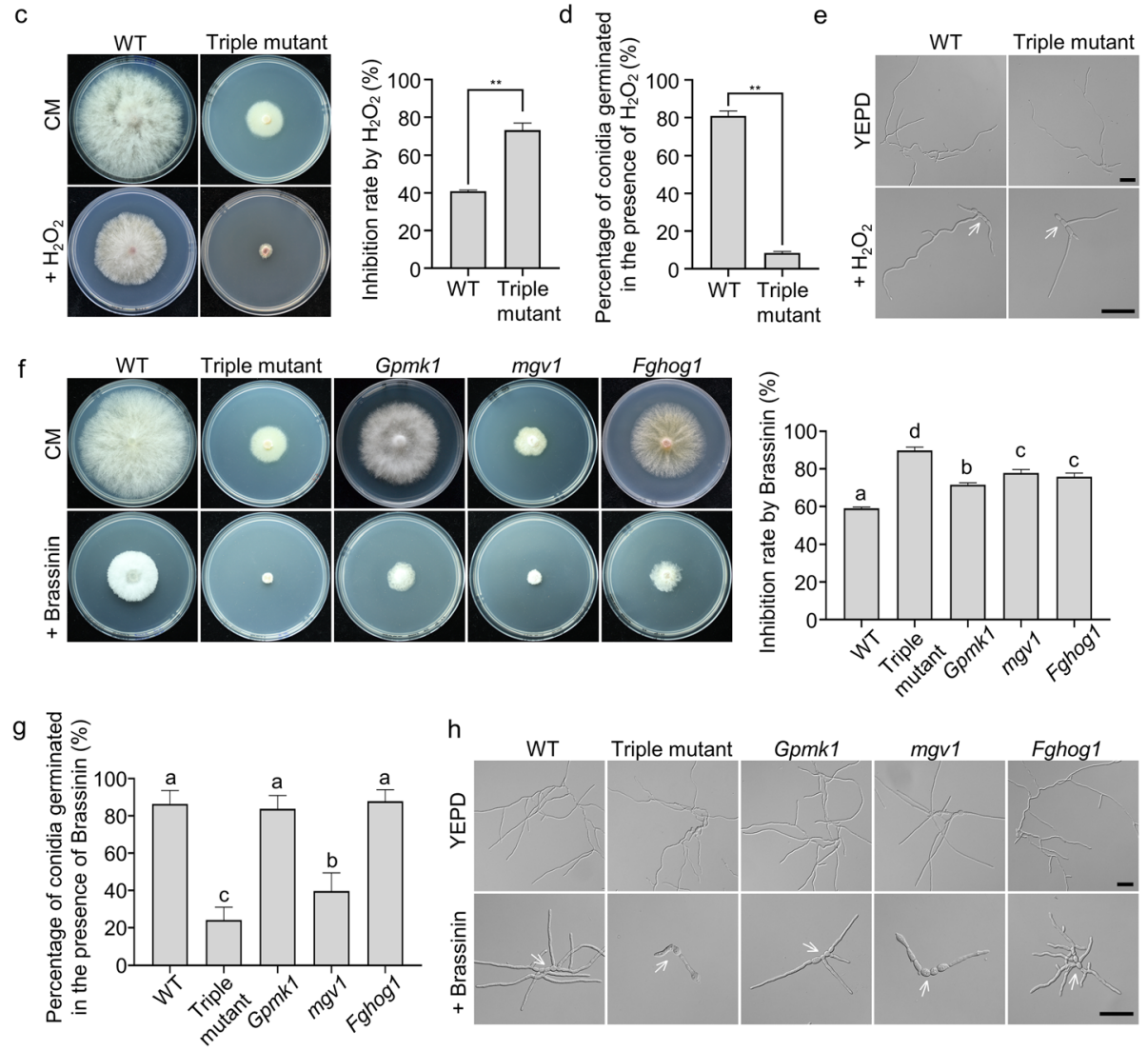

Fig. 3 The gmpk1 mgv1 Fghog1 triple mutant were defective in responses to abiotic stresses. a. Cultures of the indicated strains grown on regular CM and CM medium with $200 \mu \mathrm{g} / \mathrm{ml}$ Congo red. b. Conidia of the indicated strains were incubated for $12 \mathrm{~h}$ in YEPD with or without $0.7 \mathrm{M} \mathrm{NaCl}$. Scale bars, $50 \mu \mathrm{m}$. c. 4-day-old cultures of the wild type and triple mutant grown on $\mathrm{CM}$ with or without $0.05 \% \mathrm{H}_{2} \mathrm{O}_{2}$. The inhibition rate by $\mathrm{H}_{2} \mathrm{O}_{2}$ is estimated as the percentage of reduction in colony diameters on $\mathrm{CM}$ with $\mathrm{H}_{2} \mathrm{O}_{2}$ in comparison with regular $\mathrm{CM}$. d. Percentage of conidia germinated in YEPD medium after incubation for $6 \mathrm{~h}$. e. Conidia of the wild type and Gpmk1 mgv1 Fghog 1 triple mutant were incubated for $12 \mathrm{~h}$ in YEPD with or without $0.005 \% \mathrm{H}_{2} \mathrm{O}_{2}$. Scale bars, $50 \mu \mathrm{m}$. Conidia were indicated by arrows. $\mathbf{f}$. $\mathrm{CM}$ cultures of the indicated strains with or without $250 \mathrm{\mu M}$ brassinin. The inhibition rate by brassinin on growth in the indicated strains were estimated with colony diameters measured with 4-dayold cultures. g. Percentage of conidia germinated in YEPD medium after incubation for $6 \mathrm{~h}$. $\mathbf{h}$. Conidia of the labelled strains incubated in YEPD with or without $250 \mu \mathrm{M}$ brassinin for $24 \mathrm{~h}$. Scale bars, $50 \mu \mathrm{m}$. Conidia were indicated by arrows. Mean and standard deviation were estimated with data from at least three $(n=3)$ independent biological replicates. Different letters indicate significant differences based on ANOVA analysis followed by Duncan's multiple range test $(P=0.05)$. The asterisk indicates significant differences based on Student's $t$-test. ${ }^{* *} P<0.01$

other two MAPK mutants had conidium germination defects in the presence of brassinin although less severe than the triple mutant (Fig. 3g). Microscopic examination revealed a severe defect of germ tube growth in the triple mutant when cultured in liquid YEPD with brassinin (Fig. 3g). Germ tubes of the Gpmk1, mgv1, and
Fghog1 mutant also tended to be shorter than those of the wild type in presence of brassinin, and the $m g v 1 \mathrm{mu}-$ tant exhibited the highest sensitivity to brassinin (Fig. 3h). Therefore, Mgv1 plays a major role in regulating responses to brassinin although the Gpmk1 and FgHog1 MAPKs also have minor effects. 


\section{Mgv1 MAPK plays a major role in the fungal-bacterial interaction}

Because MAPKs are likely involved in bacterial-fungal interactions in F. graminearum (Zhang et al. 2021), we then assayed the effect of co-incubation with Bacillus velezensis strain ZQT, a biocontrol agent that inhibits pathogen growth as well as induces systemic resistance in plants (Fira et al., 2018). In antagonistic tests, the Gpmk1 mgv1 Fghog1 triple deletion mutant showed increased sensitivity to $B$. velezensis in comparison with the wild type (Fig. 4a). Treatments with B. velezensis resulted in highly vacuolated conidia and shorter germ tubes that appeared to swell at the tip in the triple mutant, which was rare in the wild type (Fig. 4b). In the presence of $B$. velezensis, abundant swollen hyphal compartments were stimulated in the triple mutant but similar irregular swellings were rarely observed in the wild type (Fig. 4c). These results suggest that B. velezensis interferes with the maintenance of polarity at germ tube or hyphal tips.

We then compared the sensitivities of the mutants deleted of individual MAPK genes to B. velezensis. Although all of them had elevated sensitivity, the mgv1 mutant was more sensitive to $B$. velezensis than the
Gpmk1 and Fghog1 mutants (Fig. 4d). Interestingly, the mgv1 mutant appeared to be more sensitive to $B$. velezensis than the Gpmk1 mgv1 Fghog1 triple mutant (Fig. 4a; 4d), suggesting that deletion of GPMK1 or FgHOG1 may partially rescues its defects in fungal-bacterial interactions.

\section{The triple mutant is defective in fungal-fungal interactions with Clonostachys rosea}

Because MAPKs have also been implicated in mycoparasitic interactions between fungi (Zhang et al. 2021), we assayed the interactions of MAPK mutants with $C$. rosea, a biocontrol agent against fungal pathogens (Sun et al., 2020a). In confrontation assays, the wild-type strain PH1 of $F$. graminearum grew faster than C. rosea strain CanS41 (Fig. 4e). Although there was a small inhibition zone at the edge of $C$. rosea colonies that were surrounded by $\mathrm{PH}-1$ hyphae, we did not observe growth of one fungus over the other (Fig. 4e). Interestingly, it appears that conidiation was stimulated in C. rosea at the edge of its colonies by PH-1 (Fig. 4e). Under the same conditions, no clear inhibition zone was observed between C. rosea and the Gpmk1 mgv1 Fghog1 triple mutant. In fact, $C$. rosea grew over colonies of the triple
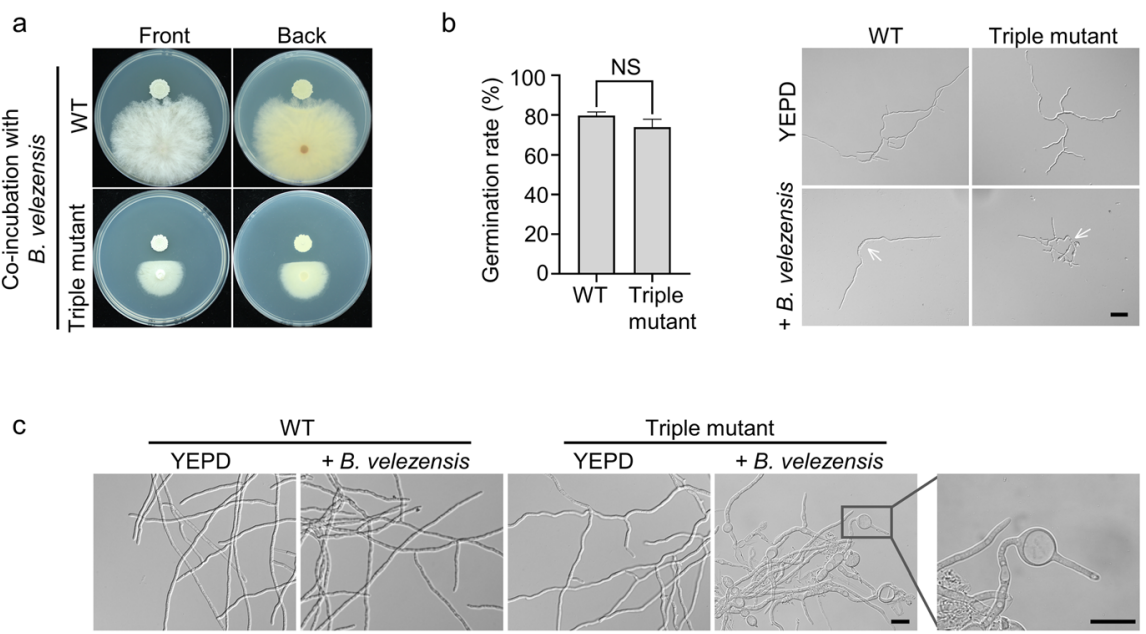

d
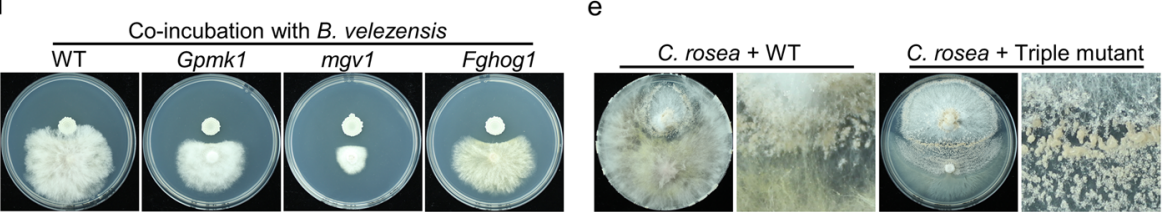

Fig. 4 Roles of MAPKs in bacterial-fungal and fungal-fungal interactions. a. Antagonistic activity of B. velezensis towards the wild type and Gpmk1 mgv1 Fghog1 triple mutant. $\mathbf{b}$. The inhibitory effects of $B$. velezensis on conidium germination $(6 \mathrm{~h})$ and germ tube growth (12 h). Scale bars, $20 \mu \mathrm{m}$. c. The formation of swollen hyphal compartments in the triple mutant stimulated by B. velezensis. Scale bars, $50 \mu \mathrm{m}$. Conidia were indicated by arrows. d. Antagonistic activity of B. velezensis towards the wild type and Gpmk1, mgv1, and Fghog1 mutants. e. 16-day-old CM confrontation-cultures of $C$. rosea (top) with the wild type or triple mutant of $F$. graminearum (bottom) with the initial contact zone amplified on the right to show stimulated conidiation in C. rosea. Mean and standard deviation were estimated with data from at least three $(n=3)$ independent biological replicates. NS indicates no significant differences based on Student's t-test 
mutant after co-incubation for 16 days and had three distinct zones. Conidiation in C. rosea was stimulated in the initial confrontation zone and the zone with overlapping growth with the triple mutant (Fig. 4e), likely due to antagonistic interactions with $F$. graminearum. In the zone beyond the triple mutant colony, hyphal growth became sparse and no stimulated conidiation was visible in C. rosea (Fig. 4e). These results suggest that the wild type, but not the triple mutant, is able to defend against mycoparasitic $C$. rosea. The defense response against $C$. rosea must be significantly weakened in the mutant deleted of all three MAPKs.

\section{Lack of MAPKs affects the expression of many ABC and MFS transporter genes}

To analyze the effects of loss of all MAPKs, we performed RNA-seq with the wild type and Gpmk1 mgv1 Fghog1 triple mutant with three biological replicates for each. In comparison with the wild type, 1469 genes and 2203 genes were up- and down-regulated over two-fold in the triple mutant, respectively (Table S2; Fig. S3). Interestingly, most of these differentially expressed genes (DEGs) were in the fast evolving subgenome associated with heterochromatins (Fig. 5a), indicating that genes regulated by these three MAPKs were subjected to fast evolution.

Based on Gene Ontology (GO)-enrichment analysis, the top 500 up-regulated DEGs in the triple mutant were not enriched in any biological process. However, the top 500 down-regulated DEGs were significantly enriched for transmembrane transport, carbohydrate transport, interspecies interactions between organisms, and oxidation-reduction process (Fig. S4).

Because transport-related genes are enriched in DEGs based on GO analysis, we examined the expression profiles of all the predicted ATP-binding cassette (ABC) and major facilitator superfamily (MFS) transporter genes in $F$. graminearum. In comparison with the wild type, 10 up-regulated and 19 down-regulated $A B C$ transporter genes were identified in the triple mutant (Fig. $5 b)$. For differentially expressed MFS transporter genes, 52 had increased transcription levels in the triple mutant whereas deletion of all three MAPKs reduced the expression of 100 MFS transporter genes (Fig. 5b). These $\mathrm{ABC}$ and MFS transporter genes are likely under transcriptional regulation by three MAPK pathways.

Interestingly, the expression level of the $F A C 1$ adenylate cyclase gene was higher in the triple MAPK mutant than the wild type (Table S2). When intracellular cAMP level was measured, the triple mutant also showed an elevated cAMP level in comparison with the wild type (Fig. 5c). These results indicated that deletion of all three MAPK genes results in the overstimulation of the cAMP-PKA signaling in F. graminearum. In contrast, three MEKK (FST11, BCK1 and SSK2) and three MEK (FST7, MKK2 and PBS2) genes had no significant changes in their expression levels in the triple mutant. The expression levels of genes encoding the $G \alpha, G \beta$, and $\mathrm{G} \gamma$ proteins also were normal in the tripe mutant, suggesting that the expression of well-conserved upstream G-proteins, MEKKs, and MEKs are not affected by deletion of these three MAPKs. In addition, deletion of three MAPKs resulted in no significant effect on the expression levels of the well-conserved STE12, MCM1, SWI6, RLM1, and ATF1 orthologs that are downstream transcription factors of MAPK signaling in the budding yeast (Jiang et al., 2018a; Zhang et al., 2021).

\section{Significant changes in metabolic profiles in the Gpmk1 mgv1 Fghog1 triple mutant}

To assay the effects of no MAPK signaling on metabolism, hyphae of the wild type and Gpmk1 mgv1 Fghog1 triple mutant were collected for widely-targeted metabolomics analysis. All three biological replicates were grouped together, indicating a high-reliability of the metabolome data (Fig. S5). A clear separation between the wild type and triple mutant were detected, suggesting the metabolite profiles in these two samples are obviously distinct (Fig. S5). A total of 2230 metabolites were identified in the metabolome of $F$. graminearum, including 1213 differentially accumulated metabolites (over two-fold changes) and 1017 metabolites with no significantly changes between the wild type and triple mutant (Fig. 5d). Among the differentially accumulated metabolites, 407 metabolites were up-regulated while 806 metabolites were down-regulated in the triple mutant (Fig. 5d). The top enriched KEGG terms among the differentially accumulated metabolites were related to arachidonic acid, linoleic acid, and alpha-linolenic acid metabolisms, and biosynthesis of terpenoid-quinone and carotenoid (Fig. $5 e)$. Interestingly, linoleic acid and $\alpha$-linolenic acid were previously reported to have antibacterial (Kusumah et al., 2020) and antifungal activities (Walters et al., 2004), which may contribute to the defects of the triple mutant in fungal-bacterial and fungal-fungal interactions.

\section{Discussion}

The roles of individual MAPKs in regulating developmental and infection processes, as well as stress responses have been characterized in a variety of fungal pathogens that differ in host ranges and infection mechanisms (Jiang et al. 2018a). However, the effect of deletion of all three MAPKs in fungal growth, differentiation, and response to biotic and abiotic stresses has not been reported in any plant pathogenic fungi or filamentous ascomycetes. In this study, we found that the Gpmk1 mgv1 Fghog1 triple mutant was viable but displayed pleiotropic defects that differ from the mgv1, Gpmk1, and Fghog1 single mutants (Fig. 
a

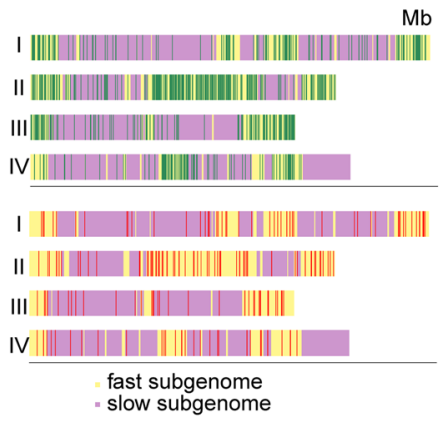

b

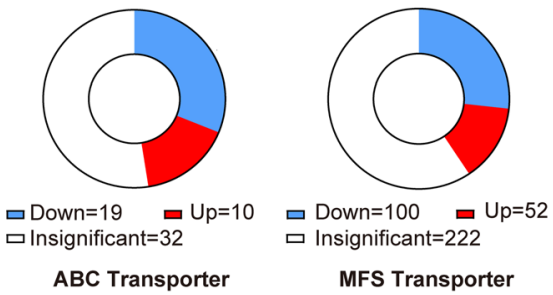

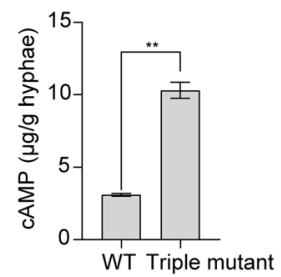

d

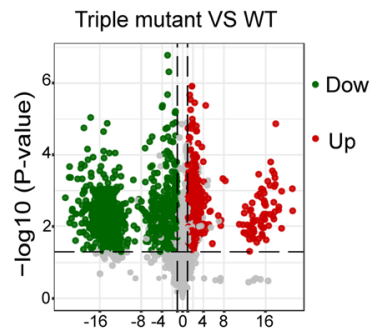

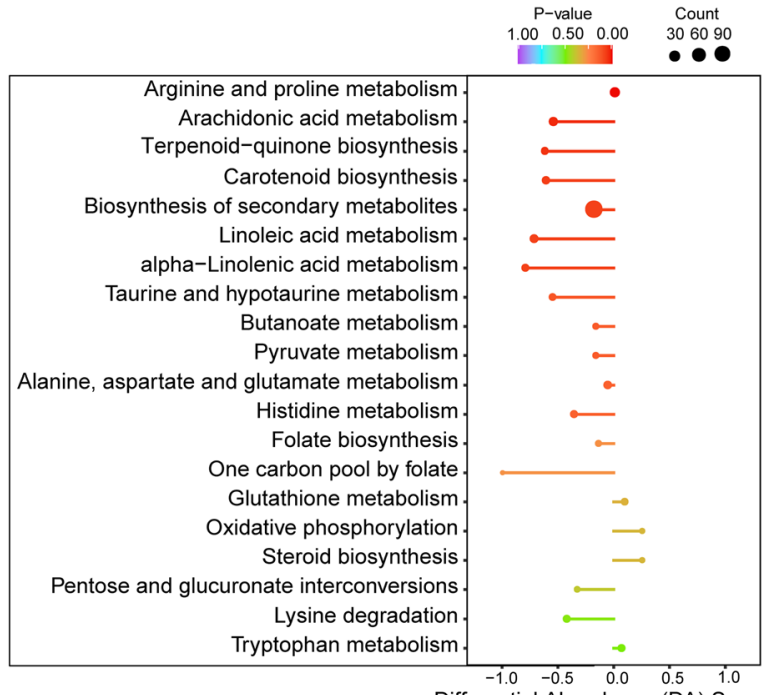

Differential Abundance (DA) Score

Fig. 5 RNA-seq analysis and metabolome profiling of the wild type and Gpmk1 mgv1 Fghog1 triple mutant. a. Distribution of up-regulated (red) and down-regulated (green) DEGs in the triple mutant on chromosomes I to IV. Each vertical line represents the chromosomal position of a specific DEG. Yellow and purple region represent the fast and slow subgenomic regions of $F$. graminearum, respectively. $\mathbf{b}$. Expression patterns of all the predicted ABC and MFS transporter genes in the wild type and triple mutant. Up and down indicate the ones that were up- or down-regulated in the triple mutant. c. The intracellular CAMP level in the wild type and triple mutant. d. A volcano plot of the 407 and 806 metabolites that were significantly increased (red dots) and decreased (green dots), respectively, in the triple mutant in comparison with the wild type. e. KEGG enrichment analysis of differentially accumulated metabolites related to listed metabolic pathways. The differential abundance (DA) score reflects the quantitative change of metabolites in the triple mutant (-, decreased; + increased) compared to the wild type. The sizes of the circles represent the number of differentially accumulated metabolites in each metabolism pathway. Distance between the circle and the center point represent the range of variation

S6). For vegetative growth, the mgv1 mutant displays severe growth defects but the Gpmk1 and Fghog1 mutants have only a minor reduction in growth rate (Hou et al. 2002; Wang et al. 2011; Zheng et al. 2012). Our earlier study has showed that null mutations in FgHOG1 partially rescued growth rate of the mgv1 mutant (Ren et al. 2019). In this study, we showed that the triple mutant grew faster than the $m g v 1$ mutant, indicating that in the absence of GPMK1, FgHOG1 is also suppressive to the growth defect caused by MGV1 deletion. However, whereas the Gpmk1 mutant had an approximately $21 \%$ reduction in growth rate compared to the wild type, deletion of GPMK1 in the mgv1 Fghog1 double mutant resulted in a 31\% decrease in growth rate (Table 1). Therefore, Gpmk1 likely contributes to the suppressive effect of FgHOG1 deletion on $m g v 1$. Gpmk1 may have overlapping functions with either Mgv1 or FgHog1 in vegetative growth. In addition, the
Gpmk1 mgv1 Fghog1 triple mutant was reduced in conidiation but had abnormal conidium morphology. Because Mgv1 is dispensable for conidiation (Hou et al. 2002), the defect of the triple mutant in conidiation is likely due to deletion of GPMK1 and FgHOG1, which is known to affect conidiation (Jenczmionka et al. 2003; Zheng et al. 2012). However, production of morphologically abnormal conidia has not been reported in any of the MAPK mutants (Hou et al. 2002; Jenczmionka and Schafer 2005; Ren et al. 2019; Wang et al. 2011; Zheng et al. 2012). It is likely that deletion of both GPMK1 and FgHOG1 is responsible for the production of longer conidia with more septa than the wild-type conidia in the triple mutant. By examining the expression levels of CON1, CON2, COM1, and HTF1 (transcription factors known to affect conidiation in F. graminearum) in our RNA-seq data, CON1 expression was affected (down-regulated 5-fold) in the triple 
mutant, likely related to the defects in conidiogenesis. In addition, deletion of three MPAKs may increase the intracellular cAMP level, which in turn activates the cAMPPKA signaling pathway. The hyper-activation of cAMPsignaling may partially rescue some defects of the MAPK triple mutant but result in additional defects in conidiogenesis.

Because all three MAPKs are important for plant infection, it is not surprising that the triple mutant was non-pathogenic in wheat head and corn silk infection assays (Fig. S6). In M. oryzae and several other plant pathogens, the PMK1 and MPS1 MAPKs are known to regulate the formation of infection structures, penetration, and growth of invasive hyphae in infected plant tissues (Jiang et al. 2018a). In F. graminearum, the triple mutant was defective in infection cushion formation, plant penetration, and invasive growth. Although GPMK1 and MGV1 MAPKs as well as their upstream kinases are known to be essential for pathogenesis in earlier studies (Hou et al. 2002; Wang et al. 2011; Yun et al. 2014), their regulatory roles in infection cushion formation and invasive growth have not been characterized. Nevertheless, the GIV1 GPCR gene that appears to function upstream of Gpmk1 is involved in regulating the development of infection cushions in F. graminearum (Jiang et al. 2019). DON is an important virulence factor that enables the fungus to spread from infected florets to the wheat rachis and other florets on the same flowering head (Bai et al., 2002; Jansen et al., 2005). The defect of the triple mutant in DON production may contribute to its defect in pathogenesis. In addition, we showed that the triple mutant was hypersensitive to phytoalexin brassinin. In Alternaria brassicicola, both AbSlt2 (Mgv1 ortholog) and AbHog1 are activated by camalexin, an indolic phytoalexin structurally related to brassinin (Joubert et al., 2011). In $F$. graminearum, the Gpmk1, mgv1 and Fghog1 mutants were all sensitive to brassinin, suggesting that these MAPKs have overlapping functions in regulating cellular responses to phytoalexins. Furthermore, our RNA-seq data showed that differentially expressed genes (DEGs) in the triple mutant were enriched in the fast evolving subgenome, which is under the control of heterochromatin and likely contains many genes important for adaption and infection (Wang et al., 2017). Some of these DEGs regulated by MAPK pathways likely play important roles in different infection processes or fungal-plant interactions.

In filamentous fungi, the CWI and HOG MAPK pathways are generally considered to be the major regulators of responses to cell wall stress and high osmolarity, respectively. While deletion of FgHOG1 was suppressive to the cell wall and growth defects of the mgv1 mutant, deletion of MGV1 slightly alleviated the hyperosmotic sensitivity of the Fghog1 mutant (Ren et al. 2019), suggesting an opposite role of MGV1 and FgHog1 in response to abiotic stressors. Nevertheless, the role of Gpmk1 and its relationship with the other two MAPKs (Mgv1 and FgHog1) in stress response are not clear in $F$. graminearum. Because the Gpmk1 mgv1 FgHog1 triple mutant displayed similar sensitivity to $\mathrm{CR}$ as the $m g v 1$ Fghog1 double mutant, indicating that Gpmk1 fails to function as a MAPK important for regulating responses to cell wall stress when MGV1 and FgHOG1 are deleted. Indeed, the role of Gpmk1 orthologs in cell wall integrity varies among different fungi. Whereas deletion of ChMK1 resulted in hypersensitivity to CR in Colletotrichun higginsianum (Wei et al., 2016), the Cfpmk1 mutant was more tolerant against CR than the wild type in C. fructicola (Liang et al., 2019). It will be important to further characterize the role of Gpmk1 in cell wall integrity and its relationship with Mgv1 and FgHog1 in $F$. graminearum. In the presence of osmotic stress, abnormal germ tubes with irregular apical and subapical swelling were observed in the triple mutant and Fghog1 mutant, but not in the mgv1 Fghog1 double mutant. It is likely that Mgv1 and Gpmk1 have opposite roles on responses to hyperosmotic stress in the absence of FgHog1. Interestingly, the Gpmk1 mutant and Gpmk1 mgv1 double mutant were also normal in germ tube morphology, indicating that Gmpk1 only plays a minor role in hyperosmotic sensitivity in the absence of FgHog1. Therefore, the increased phosphorylation level and over-activation of Gpmk1 in the mgv1 Fghog1 double mutant (Ren et al. 2019) may contribute to the suppressive effects of FgHOG1 deletion on the mgv1 mutant.

Plant pathogenic fungi such as $F$. graminearum have to interact and compete with other microbes for survival on plant debris and during plant infection (Chen et al., 2018; Zhang et al. 2021). In comparison with the roles of MAPKs in response to abiotic stresses, less is known about their functions in interactions with bacteria and other fungi. In this study, we found that the triple mutant was hypersensitive to B. velezensis (Jiang et al., 2018b). MGV1 likely plays a major role in regulating the interaction of $F$. graminearum with $B$. velezensis because the $m g v 1$ mutant appeared to be more sensitive than the triple mutant (Fig. S6). Although the Gpmk1 and Fghog1 mutants were slightly increased in sensitivity, deletion of FgHOG1 and GPMK1 in the mgv1 mutant increased its resistance against $B$. velezensis, suggesting possible crosstalk among these MAPKs in regulating fungal-bacterial interactions. Limited studies in other fungi (such as $S$. cerevisiae, $V$. dahlia and Rhizopus microspores) have showed that MAPKs can be activated by microbeassociated molecular patterns (MAMPs) or interactions with bacteria (Han et al., 2015; Lastovetsky et al., 2016; 
Marques et al., 2006). In F. graminearum, the phosphorylation of Mgv1 was inhibited by HopAI, an effector from Pseudomonas syringae (Zhang et al., 2017). Similar to their plant and animal counterparts, filamentous fungi have Nod-like immune receptors (NLRs) for signal recognition and activation of downstream targets (Uehling et al., 2017). F. graminearum has over 60 predicted NLR genes and some of them may be involved in recognizing MAMPS and fungal-bacterial interactions.

The endophytic fungus $C$. rosea is a well-known biological control agent against diverse phytopathogenic fungi (Demissie et al., 2018). In confrontation assays, the wild type $F$. graminearum was able to defend against mycoparasitic hyphae and grew surrounding $C$. rosea colonies with a small inhibition zone in their antagonistic interactions. Deletion of all three MAPKs appeared to compromise its ability to defend against C. rosea, which grew over the triple mutant and often resulted in hyphal lysis in F. graminearum after incubation for 16 days (Fig. S6). As a potent inhibitor of protein synthesis in eukaryotic organisms by binding to the large ribosome subunit, DON is an important virulence factor during plant infection and may also play an important role in antagonistic interactions with other fungi (Chen et al., 2019). The triple mutant was blocked in DON production, which may contribute to its defects in biotic interactions with $C$. rosea. In addition, the triple mutant had defects in cell wall integrity. A weakened cell wall may also affect fungal-fungal interactions. Nevertheless, the triple mutant still stimulated conidiation in C. resea, suggesting that it may still produce other antagonistic compounds. Although MAPKs are known to be related to mycoparasitism in mycoparasitic fungi (Mukherjee et al., 2003; Reithner et al., 2007; Sun et al., 2020b), their roles in fungal-fungal interactions have not been reported in any plant pathogenic fungi. Therefore, it is important to further characterize the roles of MAPK pathways and their upstream receptors in antagonistic fungal-fungal interactions in F. graminearum and other fungal pathogens.

\section{Materials \& methods}

\section{Fungal strains and culture conditions}

The wild-type $F$. graminearum strain PH-1 and MAPK mutants generated in this study were routinely cultured on potato dextrose agar (PDA). Complete medium (CM) cultures grown at $25^{\circ} \mathrm{C}$ were used for assaying growth rate and colony morphology (Wang et al. 2011). Conidiation was assayed with 5-day-old carboxymethylcellulose (CMC) cultures as described (Hou et al. 2002). For sexual reproduction, aerial hyphae of 7-day-old carrot agar cultures were pressed down and incubated at $25^{\circ} \mathrm{C}$ under black light as described (Jiang et al., 2016). Protoplast preparation and polyethylene glycol (PEG)-mediated transformation were performed as described (Hou et al. 2002). Hygromycin B, geneticin, and fludioxonil were added to the final concentration at 300,400 and $10 \mu \mathrm{g} / \mathrm{ml}$, respectively, for transformant selection.

\section{Generation of the Gpmk1 mgv1 Fghog1 triple deletion mutant}

To generate the Gpmk1 mgv1 double mutant, the $982 \mathrm{~kb}$ upstream and $690 \mathrm{~kb}$ downstream flanking sequences of MGV1 were amplified with primer pairs $M G V 1 F / 2 R$, MGV3F/4R (Table S1), respectively. The resulting PCR products were purified and connected to the neomycin resistance gene cassette by overlapping PCR and transformed into protoplasts of the Gpmk1 mutant as described (Wang et al. 2011; Zhou et al., 2011). Transformants resistant to both hygromycin and geneticin were confirmed by PCR. To generate the triple mutant, the flanking sequences of FgHOG1 were amplified with primer pairs HOG1F/2R and HOG3F/4R (Table S1), and then connected with the neomycin resistance gene cassette by overlapping PCR. The resulting PCR product was transformed into protoplasts of the Gpmk1 mgv1 double mutant. Transformants resistant to hygromycin, geneticin, and fludioxonil were verified by PCR for the deletion of FgHOG1 (Ren et al. 2019).

\section{Assays for defects in responses to abiotic stresses}

The final concentration of $200 \mu \mathrm{g} / \mathrm{ml} \mathrm{CR}, 0.05 \% \mathrm{H}_{2} \mathrm{O}_{2}$ or $250 \mu \mathrm{M}$ brassinin was added to $\mathrm{CM}$ to assay for colony growth at $25^{\circ} \mathrm{C}$ as described (Wang et al. 2011). Colony morphology was examined and photographed after incubation for 4 days. To assay for conidium germination and germ tube growth, the final concentration of $0.7 \mathrm{M}$ $\mathrm{NaCl}, 0.005 \% \mathrm{H}_{2} \mathrm{O}_{2}$ or $250 \mu \mathrm{M}$ brassinin was added to freshly harvested conidia $\left(10^{6}\right.$ spores $\left.\mathrm{ml}^{-1}\right)$ resuspended in YEPD medium. After incubation at $25^{\circ} \mathrm{C}$ for $6 \mathrm{~h}$, germination rates were counted and analyzed. After incubation at $25^{\circ} \mathrm{C}$ for $12 \mathrm{~h}$ or $24 \mathrm{~h}$, germlings were examined with an Olympus BX-51 microscope. Each experiment was repeated at least three times independently.

\section{Plant infection and DON biosynthesis assays}

Conidia of $\mathrm{PH}-1$ and the triple mutant were harvested from 5-day-old CMC cultures and resuspended to $10^{5}$ spores $\mathrm{ml}^{-1}$ in sterile double-distilled water (DDW). Wheat heads of 6-week-old cultivar XiaoYan 22 were inoculated with $10 \mu \mathrm{l}$ of conidium suspensions at the fifth spikelet from the base (Gale et al., 2007). Spikelets with typical FHB symptoms were examined at 14 dpi to estimate the disease index as described (Ding et al. 2009). Mean and standard deviation of the disease index were estimated with data from three independent replicates with at least ten wheat heads examined in each replicate. DON production in the inoculated spikelets sampled at 
14 dpi was assayed by GCMS-QP2010 with AOC-20i autoinjector (Hu et al., 2014).

To assay the formation of infection cushions, wheat lemmas inoculated with $F$. graminearum conidia were sampled at $2 \mathrm{dpi}$, fixed with $4 \%$ ( $\mathrm{vol} / \mathrm{vol}$ ) glutaraldehyde, and coated with gold-palladium before examination with a JEOL 6360 scanning electron microscope as described (Boenisch and Schafer 2011). To assay for infectious growth, the top 1-2 mm portion of wheat coleoptiles was excised and inoculated with $2 \mu$ of freshly prepared conidium suspensions $\left(10^{5}\right.$ spores $\left.\mathrm{ml}^{-1}\right)$ over the wound sites (Zhang et al., 2012). After culture at $25^{\circ} \mathrm{C}$ with a $12 \mathrm{~h}$ light $/ 12 \mathrm{~h}$ dark photoperiod for two days, inoculated seedlings were stained with Alexa Fluor 488 and examined for invasive hyphae with a Olympus FV3000 confocal microscope (Jiang et al. 2019).

\section{Assays for fungal-bacterial and fungal-fungal interaction} To assay fungal-bacterial interactions, $B$. velezensis strain ZQT (Chen et al. 2018) and F. graminearum strains (PH-1 and the MAPK mutants) were inoculated $2.5 \mathrm{~cm}$ apart on the opposite side of CM medium. After incubation at $25^{\circ} \mathrm{C}$ for 3 days, inhibition of colonial growth was examined. To assay its inhibitory effects on conidium germination and hyphal growth, $3 \mathrm{ml} B$. velezensis $(\mathrm{OD} 600=1.0)$ was mixed with fungal conidia and then resuspended in liquid YEPD $\left(10^{5}\right.$ spores $\left.\mathrm{ml}^{-1}\right)$. After incubation at $25^{\circ} \mathrm{C}$ for $6 \mathrm{~h}$ or $12 \mathrm{~h}$, germination and germlings were examined with an Olympus BX-51 microscope. Tip or intercalary swelling was observed after $24 \mathrm{~h}$ incubation. Each experiment was repeated at least three times independently.

To assay for fungal-fungal interactions, $C$. rosea strain CanS41 (Demissie et al. 2018) and F. graminearum strains were inoculated $3.5 \mathrm{~cm}$ apart on $\mathrm{CM}$ plates and incubated $25^{\circ} \mathrm{C}$. Growth of both fungi and their antagonistic interactions were observed daily or periodically for up to 16 days.

\section{RNA-seq and metabolome analyses}

Total RNAs isolated from aerial hyphae cultured on 5day-old CM medium were used for sequencing with Illumina HiSeq 2500 at Novogene Bioinformatics Technology (Beijing). RNA-seq reads were mapped to the $\mathrm{PH}-1$ reference genome using HISAT2 with its two-step algorithm (Kim et al., 2015). RNA-seq data were deposited in the NCBI SRA database under accession numbers SRR16364330-16364335. The number of reads (counts) aligned to each gene was calculated by FeatureCounts. Differentially expressed genes were identified by read count analysis with edgeRun using TMM normalization as described (Dimont et al., 2015). GO annotation was carried out with Blast2GO and GO enrichment analysis was performed by the parent-child union method with
Benjamini-Hochberg correction as developed in Ontologizer (Conesa et al., 2005).

Hyphae freshly harvested from 5-day-old CM cultures were used for metabolite profile analysis at Wuhan MetWare Biotechnology Co., Ltd. The freeze-dried samples were ground to a uniform powder prior to metabolomics analyses. The resulting samples were used for metabolite profiling with a liquid chromatography-electrospray ionization-tandem mass spectrometry (LC-ESI-MS/MS) system (HPLC, Shim-pack UFLC SHIMADZU CBM30A system; MS, Applied Biosystems 6500 Q TRAP) as described (Sun et al., 2021). Metabolite quantification was performed with multiple reaction monitoring (MRM) in triple-quadrupole mass spectrometry (Chen et al., 2013). Metabolites were identified by comparing the fragmentation patterns, retention times, and accurate $\mathrm{m} / \mathrm{z}$ values with the standards in the MetWare and public databases. The relative importance of each metabolite to the PLSDA model was checked with the parameter called variable importance in projection (VIP). Metabolites with VIP $\geq 1$ and fold change $\geq 2$ were considered as differential metabolites for group discrimination (Zhang et al., 2020). KEGG enrichment analysis of differentially accumulated metabolites was performed using KOBAS 2.0 (Xie et al., 2011).

\section{Data accessibility statement}

The data that support the findings of this study are available from the corresponding authors upon request. RNA-seq data generated in this study are accessible under accession numbers SRR16364330-16364335.

\section{Supplementary Information}

The online version contains supplementary material available at https://doi. org/10.1007/s44154-021-00025-y.

Additional file 1: Fig. S1. Generation of triple deletion mutants of MAPKs. Lane Up and lane Down showed the occurrence of homologous recombination at the upstream and downstream flanking sequences of the labelled MAPK gene, respectively. Lane T shows the deletion of labelled MAPK genes. Lane $\mathrm{H}$ or $\mathrm{G}$ showed the amplification of selectable marker genes.

Additional file 2: Fig. S2. Surface hydrophobicity assays with the wild type and triple mutant. Photos were taken 15 min after placing droplets of $20 \mu \mathrm{l}$ red ink on the colony surface.

Additional file 3: Fig. S3. A volcano plot of the 1469 up-regulated (red dots) and 2203 down-regulated (green dots) DEGs in Gpmk1 mgv1 Fghog1 triple mutant compared to the wild type.

Additional file 4: Fig. S4. GO enrichment analysis of the downregulated DEGS in the triple mutant. BP, Biological Process; CC, Cellular Components; MF, Molecular Function.

Additional file 5: Fig. S5. Multidimensional scaling plot of the metabolomic profiles of the wild type and triple mutant.

Additional file 6: Fig. S6. A proposed model for the functions and crosstalk of three MAPK pathways. a. Roles of F. graminearum MAPKs in fungal development, pathogenicity, DON production and abiotic stress responses. b. Roles of F. graminearum MAPKs in fungal-bacterial interaction and fungal-fungal interaction. 
Additional file 7: Table S1. Primers used in this study.

Additional file 8: Table S2. Profiles of the differentially expressed genes in the wild type and Gpmk1 mgv1 Fghog1 triple mutant.

\section{Acknowledgements}

We thank Drs. Hua Zhao, Fengping Yuan, Guoyun Zhang, Ping Xiang, Chengyu Gao and Jun Fan for assistance with confocal and SEM observations and DON measurement. We also thank Dr. Qiang Zhang for kindly providing bacterial strains and Drs. Qinhu Wang and Guanghui Wang for fruitful discussion.

\section{Code availability (software application or custom code)} Not applicable.

\section{Additional declarations for articles in life science journals that report} the results of studies involving humans and/or animals Not applicable.

\section{Authors' contributions}

CJ and JRX designed this project and wrote the paper. JYR, YHZ, YHW, CLL, and $X Z$ performed the experiments. JYR, CJ, ZYB and HQL analyzed the data. All authors read and approved the final manuscript.

\section{Funding}

This work was supported by grants from the National Youth Talent Support Program and National Natural Science Foundation of China (no. 31772114) to JC and grants from NSWBSI to JRX.

\section{Availability of data and materials}

All data generated or analyzed during this study are included in this published article and its supplementary information files.

\section{Declarations}

Ethics approval and consent to participate

All authors consent to participate.

\section{Consent for publication}

All authors consent for publication.

\section{Competing interests}

Author JRX is a member of the Editorial Board, but was not involved in the journal's review of, or decisions related to, this manuscript.

Received: 1 November 2021 Accepted: 9 December 2021

Published online: 17 January 2022

\section{References}

Audenaert K, Vanheule A, Hofte M, Haesaert G (2013) Deoxynivalenol: a major player in the multifaceted response of fusarium to its environment. Toxins (Basel) 6(1):1-19. https://doi.org/10.3390/toxins6010001

Bai GH, Desjardins AE, Plattner RD (2002) Deoxynivalenol-nonproducing Fusarium graminearum causes initial infection, but does not cause disease spread in wheat spikes. Mycopathologia 153(2):91-98. https://doi.org/10.1023/a:101441 9323550

Bermejo C, Rodriguez E, Garcia R, Rodriguez-Pena JM, de la Concepcion MLR, Rivas C et al (2008) The sequential activation of the yeast HOG and SLT2 pathways is required for cell survival to cell wall stress. Mol Biol Cell 19:11131124. https://doi.org/10.1091/mbc.E07-08-0742

Boenisch MJ, Schafer W (2011) Fusarium graminearum forms mycotoxin producing infection structures on wheat. BMC Plant Biol 11(1):110. https://doi.org/10.1186/1471-2229-11-110

Chen W, Gong L, Guo ZL, Wang WS, Zhang HY, Liu XQ, Yu S, Xiong L, Luo J (2013) A novel integrated method for large-scale detection, identification, and quantification of widely targeted metabolites: application in the study of rice metabolomics. Mol Plant 6(6):1769-1780. https://doi.org/10.1093/mp/sst080

Chen Y, Kistler HC, Ma ZH (2019) Fusarium graminearum trichothecene mycotoxins: biosynthesis, regulation, and management. Annu Rev Phytopathol 57(1):15-39. https://doi.org/10.1146/annurev-phyto-082718-100318
Chen Y, Wang J, Yang N, Wen ZY, Sun XP, Chai YR, Ma Z (2018) Wheat microbiome bacteria can reduce virulence of a plant pathogenic fungus by altering histone acetylation. Nat Commun 9(1):3429. https://doi.org/10.1038/ s41467-018-05683-7

Conesa A, Gotz S, Garcia-Gomez JM, Terol J, Talon M, Robles M (2005) Blast2GO: a universal tool for annotation, visualization and analysis in functional genomics research. Bioinformatics 21(18):3674-3676. https://doi.org/10.1093/ bioinformatics/bti610

Demissie ZA, Foote SJ, Tan YF, Loewen MC (2018) Profiling of the transcriptomic responses of Clonostachys rosea upon treatment with fusarium graminearum Secretome. Front Microbiol 9:1061. https://doi.org/10.3389/fmicb.2018.01061

Dilks T, Halsey K, De Vos RP, Hammond-Kosack KE, Brown NA (2019) Non-canonical fungal G-protein coupled receptors promote fusarium head blight on wheat. PLoS Pathog 15(4):e1007666. https://doi.org/10.1371/journal.ppat.1007666

Dimont E, Shi JT, Kirchner R, Hide W (2015) edgeRun: an R package for sensitive, functionally relevant differential expression discovery using an unconditional exact test. Bioinformatics 31(15):2589-2590. https://doi.org/10.1093/ bioinformatics/btv209

Ding SL, Mehrabi R, Koten C, Kang ZS, Wei YD, Seong KY, Kistler HC, Xu JR (2009) Transducin Beta-like gene FTL1 is essential for pathogenesis in fusarium graminearum. Eukaryot Cell 8(6):867-876. https://doi.org/10.1128/Ec.00048-09

Fira D, Dimkic I, Beric T, Lozo J, Stankovic S (2018) Biological control of plant pathogens by Bacillus species. J Biotechnol 285:44-55. https://doi.org/10.101 6/j.jbiotec.2018.07.044

Gale LR, Ward TJ, Balmas V, Kistler HC (2007) Population subdivision of fusarium graminearum sensu stricto in the upper midwestern United States. Phytopathology 97(11):1434-1439. https://doi.org/10.1094/PHYTO-97-11-1434

Goswami RS, Kistler HC (2004) Heading for disaster: fusarium graminearum on cereal crops. Mol Plant Pathol 5(0036):515-525. https://doi.org/10.1111/j.13 64-3703.2004.00252.x

Gu Q, Chen Y, Liu Y, Zhang C, Ma Z (2015) The transmembrane protein FgShol regulates fungal development and pathogenicity via the MAPK module Ste50-Ste11-Ste7 in Fusarium graminearum. New Phytol 206(1):315-328. https://doi.org/10.1111/nph.13158

Han Q, Wu F, Wang X, Qi H, Shi L, Ren A, Liu Q, Zhao M, Tang C (2015) The bacterial lipopeptide iturins induce Verticillium dahliae cell death by affecting fungal signalling pathways and mediate plant defence responses involved in pathogen-associated molecular pattern-triggered immunity. Environ Microbiol 17(4):1166-1188. https://doi.org/10.1111/1462-2920.12538

Harris LJ, Balcerzak M, Johnston A, Schneiderman D, Ouellet T (2016) Hostpreferential fusarium graminearum gene expression during infection of wheat, barley, and maize. Fungal Biol 120(1):111-123. https://doi.org/10.1016/ j.funbio.2015.10.010

Hou ZM, Xue CY, Peng YL, Katan T, Kistler HC, Xu JR (2002) A mitogen-activated protein kinase gene (MGV1) in fusarium graminearum is required for female fertility, heterokaryon formation, and plant infection. Mol Plant Microbe In 15(11):1119-1127. https://doi.org/10.1094/Mpmi.2002.15.11.1119

Hu S, Zhou X, Gu X, Cao S, Wang C, Xu JR (2014) The cAMP-PKA pathway regulates growth, sexual and asexual differentiation, and pathogenesis in fusarium graminearum. Mol Plant-Microbe Interact 27(6):557-566. https://doi. org/10.1094/MPMI-10-13-0306-R

Jansen C, von Wettstein D, Schäfer W, Kogel KH, Felk A, Maier FJ (2005) Infection patterns in barley and wheat spikes inoculated with wild-type and trichodiene synthase gene disrupted fusarium graminearum. Proc Natl Acad Sci U S A 102(46):16892-16897. https://doi.org/10.1073/pnas.0508467102

Jenczmionka NJ, Maier FJ, Losch AP, Schafer W (2003) Mating, conidiation and pathogenicity of fusarium graminearum, the main causal agent of the headblight disease of wheat, are regulated by the MAP kinase gpmk1. Curr Genet 43(2):87-95. https://doi.org/10.1007/s00294-003-0379-2

Jenczmionka NJ, Schafer W (2005) The Gpmk1 MAP kinase of fusarium graminearum regulates the induction of specific secreted enzymes. Curr Genet 47(1):29-36. https://doi.org/10.1007/s00294-004-0547-z

Jiang C, Cao S, Wang Z, Xu H, Liang J, Liu H, Wang G, Ding M, Wang Q, Gong C, Feng C, Hao C, Xu JR (2019) An expanded subfamily of Gprotein-coupled receptor genes in fusarium graminearum required for wheat infection. Nat Microbiol 4(9):1582-1591. https://doi.org/10.1038/s41 564-019-0468-8

Jiang C, Zhang C, Wu C, Sun P, Hou R, Liu H, Wang C, Xu JR (2016) TRI6 and TRI10 play different roles in the regulation of deoxynivalenol (DON) production by CAMP signalling in fusarium graminearum. Environ Microbiol 18(11):3689-3701. https://doi.org/10.1111/1462-2920.13279 
Jiang C, Zhang X, Liu H, Xu JR (2018a) Mitogen-activated protein kinase signaling in plant pathogenic fungi. PLoS Pathog 14(3):e1006875. https://doi.org/10.13 71/journal.ppat.1006875

Jiang CH, Liao MJ, Wang HK, Zheng MZ, XU JJ, Guo JH (2018b) Bacillus velezensis, a potential and efficient biocontrol agent in control of pepper gray mold caused by Botrytis cinerea. Biol Control 126:147-157. https://doi.org/10.1016/ j.biocontrol.2018.07.017

Joubert A, Bataille-Simoneau N, Campion C, Guillemette T, Hudhomme P, lacomiVasilescu B, Leroy T, Pochon S, Poupard P, Simoneau P (2011) Cell wall integrity and high osmolarity glycerol pathways are required for adaptation of Alternaria brassicicola to cell wall stress caused by brassicaceous indolic phytoalexins. Cell Microbiol 13(1):62-80. https://doi.org/10.1111/j.1462-5822.2 010.01520.x

Kim D, Langmead B, Salzberg SL (2015) HISAT: a fast spliced aligner with low memory requirements. Nat Methods 12(4):357-360. https://doi.org/10.1038/ nmeth.3317

Kusumah D, Wakui M, Murakami M, Xie XN, Yukihito K, Maeda I (2020) Linoleic acid, alpha-linolenic acid, and monolinolenins as antibacterial substances in the heat-processed soybean fermented with Rhizopus oligosporus. Biosci Biotechnol Biochem 84(6):1285-1290. https://doi.org/10.1080/09168451.202 0.1731299

Lastovetsky OA, Gaspar ML, Mondo SJ, LaButti KM, Sandor L, Grigoriev IV et al (2016) Lipid metabolic changes in an early divergent fungus govern the establishment of a mutualistic symbiosis with endobacteria. Proc Natl Acad Sci U S A 113(52):15102-15107. https://doi.org/10.1073/pnas.1615148113

Li G, Zhou X, Xu JR (2012) Genetic control of infection-related development in Magnaporthe oryzae. Curr Opin Microbiol 15(6):678-684. https://doi.org/10.1 016/j.mib.2012.09.004

Liang XF, Wei TY, Cao MY, Zhang X, Liu WK, Kong YY, Zhang R, Sun G (2019) The MAP kinase CFPMK1 is a key regulator of pathogenesis, development, and stress tolerance of Colletotrichum fructicola. Front Microbiol 10:1070. https:// doi.org/10.3389/fmicb.2019.01070

Marques JM, Rodrigues RJ, de Magalhaes-Sant'Ana AC, Goncalves T (2006) Saccharomyces cerevisiae Hog1 protein phosphorylation upon exposure to bacterial endotoxin. J Biol Chem 281(34):24687-24694. https://doi.org/10.1 074/jbc.M603753200

Mukherjee PK, Latha J, Hadar R, Horwitz BA (2003) TmkA, a mitogen-activated protein kinase of Trichoderma virens, is involved in biocontrol properties and repression of conidiation in the dark. Eukaryot Cell 2(3):446-455. https://doi. org/10.1128/EC.2.3.446-455.2003

Posas F, Takekawa M, Saito H (1998) Signal transduction by MAP kinase cascades in budding yeast. Curr Opin Microbiol 1(2):175-182. https://doi.org/10.1016/ s1369-5274(98)80008-8

Reithner B, Schuhmacher R, Stoppacher N, Pucher M, Brunner K, Zeilinger S (2007) Signaling via the Trichoderma atroviride mitogen-activated protein kinase Tmk 1 differentially affects mycoparasitism and plant protection. Fungal Genet Biol 44(11):1123-1133. https://doi.org/10.1016/j.fgb.2007.04.001

Ren JY, Li CL, Gao CY, Xu JR, Jiang C, Wang GH (2019) Deletion of FgHOG1 is suppressive to the mgv1 mutant by stimulating Gpmk1 activation and avoiding intracellular turgor elevation in fusarium graminearum. Front Microbiol 10:1073. https://doi.org/10.3389/fmicb.2019.01073

Sellam A, lacomi-Vasilescu B, Hudhomme P, Simoneau P (2007) In vitro antifungal activity of brassinin, camalexin and two isothiocyanates against the crucifer pathogens Alternaria brassicicola and Alternaria brassicae. Plant Pathol 56(2): 296-301. https://doi.org/10.1111/j.1365-3059.2006.01497.x

Sun T, Zhang J, Zhang Q, Li X, Li M, Yang Y, Zhou J, Wei Q, Zhou B (2021) Transcriptome and metabolome analyses revealed the response mechanism of apple to different phosphorus stresses. Plant Physiol Biochem 167:639650. https://doi.org/10.1016/j.plaphy.2021.08.040

Sun ZB, Li SD, Ren Q, Xu JL, Lu X, Sun MH (2020a) Biology and applications of Clonostachys rosea. J Appl Microbiol 129(3):486-495. https://doi.org/10.1111/ jam.14625

Sun ZB, Wang Q, Sun MH, Li SD (2020b) The mitogen-activated protein kinase gene Crmapk is involved in Clonostachys chloroleuca mycoparasitism. Mol Plant Microbe In 33:902-910. https://doi.org/10.1094/Mpmi-03-20-0062-R

Uehling J, Deveau A, Paoletti M (2017) Do fungi have an innate immune response? An NLR-based comparison to plant and animal immune systems. PLoS Pathog 13(10):e1006578. https://doi.org/10.1371/journal.ppat.1006578

Urban M, Mott E, Farley T, Hammond-Kosack K (2003) The fusarium graminearum MAP 1 gene is essential for pathogenicity and development of perithecia. Mol Plant Pathol 4:347-359. https://doi.org/10.1046/J.1364-3703.2003.00183.X
Walters D, Raynor L, Mitchell A, Walker R, Walker K (2004) Antifungal activities of four fatty acids against plant pathogenic fungi. Mycopathologia 157(1):87-90 https://doi.org/10.1023/b:myco.0000012222.68156.2c

Wang CF, Zhang SJ, Hou R, Zhao ZT, Zheng Q, Xu QJ, Zheng D, Wang G, Liu H, Gao X, Ma JW, Kistler HC, Kang Z, Xu JR (2011) Functional analysis of the Kinome of the wheat scab fungus fusarium graminearum. PLoS Pathog 7(12): e1002460. https://doi.org/10.1371/journal.ppat.1002460

Wang G, Li G, Zhang S, Jiang C, Qin J, Xu JR (2015) Activation of the signalling mucin MoMsb2 and its functional relationship with Cbp1 in Magnaporthe oryzae. Environ Microbiol 17(8):2969-2981. https://doi.org/10.1111/1462-292 0.12847

Wang QH, Jiang C, Wang CF, Chen CJ, Xu JR, Liu HQ (2017) Characterization of the two-speed subgenomes of fusarium graminearum reveals the fast-speed subgenome specialized for adaption and infection. Front Plant Sci 8:140. https://doi.org/10.3389/fpls.2017.00140

Wei W, Xiong Y, Zhu WJ, Wang NC, Yang GG, Peng F (2016) Colletotrichum higginsianum mitogen-activated protein kinase ChMK1: Rolein growth, Cell Wall integrity, Colony Melanization, and pathogenicity. Front Microbiol 7: 1212. https://doi.org/10.3389/fmicb.2016.01212

Xie C, Mao X, Huang J, Ding Y, Wu J, Dong S, Kong L, Gao G, Li CY, Wei L (2011) KOBAS 2.0: a web server for annotation and identification of enriched pathways and diseases. Nucleic Acids Res 39(suppl_2):W316-W322. https:// doi.org/10.1093/nar/gkr483

Xu JR, Staiger CJ, Hamer JE (1998) Inactivation of the mitogen-activated protein kinase Mps1 from the rice blast fungus prevents penetration of host cells but allows activation of plant defense responses. Proc Natl Acad Sci U S A 95(21): 12713-12718. https://doi.org/10.1073/pnas.95.21.12713

Yun Y, Liu Z, Zhang J, Shim WB, Chen Y, Ma Z (2014) The MAPKK FgMkk1 of fusarium graminearum regulates vegetative differentiation, multiple stress response, and virulence via the cell wall integrity and high-osmolarity glycerol signaling pathways. Environ Microbiol 16(7):2023-2037. https://doi. org/10.1111/1462-2920.12334

Zhang Q, Wang LL, Liu ZG, Zhao ZH, Zhao J, Wang ZT et al (2020) Transcriptome and metabolome profiling unveil the mechanisms of Ziziphus jujuba Mill pee coloration. Food Chem 312:125903. https://doi.org/10.1016/j.foodchem.201 9.125903

Zhang X, Liu W, Li Y, Li G, Xu JR (2017) Expression of HopAl interferes with MAP kinase signalling in Magnaporthe oryzae. Environ Microbiol 19(10):4190-4204. https://doi.org/10.1111/1462-2920.13884

Zhang X, Wang Z, Jiang C, Xu JR (2021) Regulation of biotic interactions and responses to abiotic stresses by MAP kinase pathways in plant pathogenic fungi. Stress Biol 1(1). https://doi.org/10.1007/s44154-021-00004-3

Zhang XW, Jia LJ, Zhang Y, Jiang G, Li X, Zhang D, Tang WH (2012) In planta stage-specific fungal gene profiling elucidates the molecular strategies of fusarium graminearum growing inside wheat coleoptiles. Plant Cell 24(12): 5159-5176. https://doi.org/10.1105/tpc.112.105957

Zheng D, Zhang S, Zhou X, Wang C, Xiang P, Zheng Q, Xu JR (2012) The FgHOG1 pathway regulates hyphal growth, stress responses, and plant infection in fusarium graminearum. PLoS ONE 7(11):e49495. https://doi.org/1 0.1371/journal.pone.0049495

Zhou X, Li G, XU JR (2011) Efficient approaches for generating GFP fusion and epitope-tagging constructs in filamentous fungi. Methods Mol Biol 722:199212. https://doi.org/10.1007/978-1-61779-040-9_15

\section{Publisher's Note}

Springer Nature remains neutral with regard to jurisdictional claims in published maps and institutional affiliations. 